EN LA MIRA: Cambios en el trabajo 



\title{
Cambios en la organización del trabajo tradicional: Conflictos y actores laborales
}

\author{
Lucena, Héctor*
}

\section{Resumen}

Este artículo forma parte de una línea de investigación más general que se ocupa de analizar las transformaciones en las Relaciones de Trabajo. El presente documento centra su atención en los cambios que experimenta la Organización del Trabajo y como ellos repercuten en la conflictividad laboral. Se parte de reconocer que la relación capital-trabajo es de naturaleza conflictiva, pero que los mecanismos de convivencia entre las partes y todo el marco regulatorio, permiten y facilitan el desenvolvimiento y la convivencia productiva. Sin embargo, los cambios en la Organización del Trabajo que reducen la fuerza de trabajo y lesionan conquistas históricas de los trabajadores, estimulan el desarrollo de conflictos colectivos, los que materializan en tanto existan constituidos los actores laborales. En el proceso de investigación seguido en esta línea, se revisa la literatura pertinente, se analizan casos de la realidad laboral venezolana reciente vía hemeroteca y entrevistas, privilegiándose la huelga de Sidor en el 2004. Se concluye, destacando que los cambios que paulatinamente se emprenden desde la empresa para modificar la Organización del Trabajo, erosionan la capacidad de respuesta de los actores laborales representativos de los trabajadores.

Palabras clave: Conflicto laboral, conflicto colectivo, conflicto individual, organización del trabajo, actores laborales.

\section{Changes in traditional work organization, labor conflicts and actors}

\section{Abstract}

This article is part of a more general line of research oriented towards analyzing transformations in labor relations. This paper focuses its attention on changes undergone in labor organization and how these changes have affected labor conflictivity. The study begins with the recognition tnat the labor-capital relationship is conflictive by nature, but that mechanisms of co-existence between the these elements and the entire regulatory framework, permit and facilitate the productive function and coexistence. However, changes in the organization of labor that reduce the work force and debilitate

\section{Recibido: 04-06-09. Aceptado: 05-01-27}

Coordinador del Doctorado en Ciencias Sociales, Mención Estudios del Trabajo, Universidad de Carabobo. E-mail: hlucena@postgrado.uc.edu.ve 
historical advances for workers, stimulate the development of collective conflicts which materialize when labor forces are constituted. In the research process in this area, pertinent literature was reviewed, including cases of recent Venezuelan labor problems, through pertinent literature and interviews, focusing especially on the Sidor strike of 2004. The conclusions point out that changes, even when gradual, undertaken by companies in order to modify work organization, erode the response capacity of the actors who represent workers.

Key words: Labor conflict, collective conflict, individual conflict, labor organization, labor unions, labors actors.

\section{Introducción}

Nos estimula elaborar una contribución sobre el tema del conflicto laboral. La Gerencia, y aún más los contenidos de los estudios gerenciales, tienden a percibir los conflictos como un fenómeno patológico. Algo así como una enfermedad social. Lo entendemos más bien como un elemento propio de sociedades con intereses contradictorios. Conflicto es controversia. Diferencias en torno a asuntos que interesan a varias partes. La convivencia productiva, que es obviamente lo cotidiano en las organizaciones, supone conflictos y consensos. Por supuesto, no se elaboran los objetivos de una y otra parte actuantes en el escenario productivo, fijando como meta el conflicto en si mismo. El capital aspira rentabilidad y acumulación. El trabajo aspira una retribución y condiciones de trabajo que le permiten mejorar su nivel de vida, así como el de sus dependientes. El conflicto, al igual que el consenso, se construye. No viene de la nada, viene de las relaciones sociales, de cómo ellas funcionen.

El discurso unificante, armonizador es una constante en la gerencia, de cualquier lugar del mundo (Lucena, 2003; Hernández, 2003; Coller, 1997). Lo que importa para el análisis, es ver la correspondencia entre este discurso y las prác- ticas productivas y administrativas, que conforman el intercambio entre la gerencia y los trabajadores. El discurso unificante es entendible, ya que se trata de una convivencia productiva. Alcanzar las metas y objetivos de la gerencia, requiere organizar los recursos hacia los fines e índices preestablecidos. La historia de la gestión siempre ha ido acompañada del discurso unificante de los intereses de empresa y trabajadores (Coller, 1997). Pero, no obstante, las mismas sociedades han visto la necesidad de permisar la acción conflictiva. Por eso el derecho a la huelga está generalizado en casi todo el mundo. De ahí el énfasis en llevar el análisis hacia el propio campo de los intereses de las partes, hacia el reconocimiento abierto de la bilateralidad, que es como entendemos se debe enfocar el fenómeno de los conflictos del trabajo.

\section{Raíces del conflicto laboral: ¿patológico o connatural?}

Las Relaciones de Trabajo como campo disciplinario que atiende el estudio de las relaciones entre capital y trabajo, tiene entre sus objetos de análisis el tema de los conflictos laborales. Lo que plantea un reto clave en el mundo del trabajo. El conflicto es una institución o proceso que utilizan las partes para contri- 
buir a la conquista de sus objetivos. Es un hecho connatural a la relación de trabajo. Es un medio de presión que coadyuva al alcance de objetivos previamente determinados. Incluso, se entiende que el sólo anuncio de un conflicto, es ya en si mismo una arma de presión. Las partes anuncian sus armas de presión con fines disuasivos, en un primer momento.

La dimensión del conflicto que interesa a las Relaciones de Trabajo, no se circunscribe sólo a los denominados legales. Se entiende más bien, que cuando las partes acuden a las instancias públicas -por ejemplo la Administración del Trabajo- para plantear una demanda con carácter conflictivo, igualmente cuando acuden a la Administración de Justicia, no es el inicio de la situación conflictiva misma. Ella viene, con toda seguridad, planteándose previamente en el marco de las relaciones directas. Hay desavenencias, que no obstante el intercambio directo entre las partes, que suele ser la instancia primaria de atención a las controversias, no pudieron ser satisfechas o resueltas. Alfonso (1967:426) en su clásico "Estudio Analítico de Ley del Trabajo Venezolana" resalta "la huelga y el lock-out no son los conflictos propiamente, sino tan sólo el desenlace de los mismos".

Los conflictos no siempre son abiertos, ruidosos, estridentes. También hay manifestaciones solapadas, larvadas en el tejido laboral que cotidianamente se construye en los espacios productivos. Desde el lado de la empresa la acción conflictiva abierta no es relevante. Se correspondería con los cierres de empresas, llamados también lock-out. Son infrecuentes. Es una medida extrema, que implica desmontar esfuerzos productivos construidos a lo largo del tiempo, y además genera dificultades con las autoridades laborales. Las que son reticentes a admitir estas situaciones, ya que suponen contratiempos con aquellos colectivos que ahí laboran, además de los suplidores y prestadores de servicios externos. En muchos casos, acuden a las instancias públicas para pedir auxilios e intervenciones ${ }^{1}$.

Hay quienes señalan que el mismo contrato de trabajo, y la subordinación del trabajador asalariado que lleva implícita, ya es una manifestación de conflictividad (Velasco, 1976). Aún más clásico, la visión marxista ubica la condición misma de asalariado como conflictiva en si misma, ya que en su seno alberga la explotación del trabajo humano. El meollo del problema es la consideración de la "fuerza de trabajo" como mercancía. Se compra y se vende. La empresa la compra y la administra. La remunera y obtiene de ella un servicio o producto. La compra por toda una jornada, y exigirá del trabajador que siga trabajando más allá del limite en el cual la empresa ya recibió el valor de lo que le cuesta: "tiempo de trabajo necesa-

1 En los últimos años, ha sido emblemático el caso de Venepal, durante varias décadas un importante emporio de la industria papelera venezolana. En los últimos tres años se vino a menos. En sus mejores momentos llegó a tener más de dos mil quinientos trabajadores en su nómina, en un conglomerado de varias plantas industriales relacionadas con este sector productivo. En la medida que la situación de crisis de funcionamiento se ha agudizado, la recurrencia al Estado aparece como el eslabón salvador. Al final, declarada la quiebra, el Estado la expropió. 
rio" y "tiempo de trabajo excedente" (Marx, 1973:147). También puede ocurrir lo contrario, que la remunera y termina la jornada y aún la empresa no ha logrado resarcir dicho costo. Que ocurre en estos casos, sencillamente la despide -la fuerza de trabajo-. De todas maneras, la jornada de la cual nos ocupamos, no es sólo en la dimensión diaria, sino en lapsos más prolongados, digamos anuales por ejemplo. Es el lapso más usual de la planificación, de la gestión y de realizar los balances. A lo largo de un año, hay períodos de baja y de alta demanda. Además las organizaciones, expresan en cualquier momento dificultades, y lo que usualmente ocurre, es mirar hacia la fuerza de trabajo, y expulsar cuantos sean necesarios para sobrevivir a la contingencia. Por lo demás el contrato temporal, es otro artilugio del cual dispone la administración empresarial.

Cabe preguntarse, si solamente el conflicto laboral ocurre en las relaciones de producción, en donde el capitalista se apropia del excedente producido por el trabajador. Es decir, en nuestras sociedades capitalistas. Aquí caben dos referencias que nos obligan a ver otras dimensiones en el origen del problema, lo que por supuesto nos amplifica la reflexión. Primero, por un lado en sociedades socialistas han visto, no obstante las limitaciones con las cuales se podía organizar la acción sindical abierta -regímenes de sindi- calismo único-, olas de protestas y conflictos laborales por demandas económicas y políticas. Recuérdese la década de los ochenta en los antiguos países socialistas europeos del este. La segunda referencia, son las acciones conflictivas laborales ante los servicios de propiedad pública y administrados igualmente por el Estado. En todas nuestras sociedades latinoamericanas, son, sin duda, en los últimos años los escenarios más frecuentes de acciones conflictivas colectivas laborales $^{2}$. Entendemos que el Estado no acumula con el "tiempo de trabajo excedente". Se señala que la esencia del conflicto está en la existencia de la autoridad y no en el sistema de producción, por eso se observa tanto en los sistemas capitalistas, como socialistas, así como en las propiedades estatales y cooperativas (Van der Laat, 1979:18).

El más emblemático de los conflictos laborales es la acción huelgaria. Es una acción colectiva abierta. Es la que más repercute en la sociedad. Mucho más si se trata de organizaciones grandes y emblemáticas por su producción, tipo de servicio y efectos en el tejido productivo y en la sociedad en general. Los conflictos colectivos en el ámbito de los servicios públicos tienen efectos multiplicadores, ya que afectan directamente el desenvolvimiento de los ciudadanos.

2 Para el caso venezolano, Antonio Romero realizó dos estudio que analizan detenidamente el fenómeno, el primero en el período 1989-1993 (1997), y el segundo en el 1994-1998 (2001). Para el caso latinoamericano ver Zapata (2003:386). 


\section{La importancia de identificar el conflicto como individual o colectivo}

En la normativa laboral y en la literatura especializada ha predominado la tendencia a colocar los conflictos individuales y los colectivos, en dos dimensiones separadas (Córdova, 2004; Van der Laat, 1979:24. Alfonso, 1967:428429; Sappia, 2002:11). Se entiende que el tratamiento que dispensan las autoridades públicas a los conflictos, ha contribuido a esta división. Se afirma que los individuales son aquellos conflictos justiciables, que también son llamados de interpretación. Los atiende la justicia laboral -los tribunales del trabajo-. En cambio los colectivos son aquéllos también llamados de intereses, y los atiende la administración del trabajo. Por supuesto ambos, son en primer lugar objeto de atención por la acción directa de las partes.

La identificación como conflicto de intereses, sólo en los casos de los económicos se presta a confusión. En realidad todos los conflictos conllevan elementos económicos. Cuando se dirime la interpretación de una norma, ya que el asunto es controversial, sin lugar a dudas, es porque en su interior hay implicaciones económicas. Así que el asunto del interés, pudiera ser más bien un hecho cuasi universal en los conflictos ${ }^{3}$.

Por lo demás, en la medida que se fomente la autorregulación del conflicto, el Estado jugaría un papel menos presencial en la solución y tratamiento de los conflictos, aunque no menos importante. Es decir, así como las partes han alcanzado un grado de madurez en la construcción regulatoria, por la vía de los contratos, convenios y negociaciones en general, es lógico admitir que una evolución avanzada de convivencia productiva, les lleve a construir igualmente los mecanismos e instituciones para dirimir sus diferencias, sean estas individuales o colectivas, de intereses o de interpretación (Sappia, 2002). Así se expresa en los convenios que crean instituciones bipartitas o tripartitas, para atender de primera mano las controversias entre las partes ${ }^{4}$. Estas experiencias conviene recomendarla, ya que en la dimensión de la OT, y los constantes cambios que se promueven en su ámbito, encuentran en la acción directa y de los mecanismos que las partes construyan, la atención a los conflictos individuales y colectivos, así como de intereses y de interpretación.

3 Alfonso (1967:433) admite esta situación al referirse “..imprecisión de las zonas fronterizas entre las dos competencias". Además se observa en la región americana varios países en donde hay un solo organismo para atender los diversos tipos de conflictos laborales, tales son los casos de Brasil, México, Panamá y Canadá (Sappia, 2002:11).

4 En Venezuela, se han establecido en los convenios colectivos de los sectores siderúrgicos y educativos. 


\section{La Organización del Trabajo, el Conflicto y la acción de los Actores Laborales en los casos de trabajadores centrales y periféricos}

Se plantea analizar si las nuevas formas de Organización del Trabajo -OT¿Suponen nuevas formas de conflictos? ¿Cuánto dificultan los conflictos las nuevas formas de OT? Trabajadores de la empresa central y trabajadores de las contratistas: ¿tienen intereses convergentes o divergentes?

Cuando nos referimos a la OT, se trata de lo siguiente: "El conjunto de aspectos técnicos y sociales que intervienen en la producción de determinado objeto o servicio. Se refiere a la división del trabajo entre las personas, así como entre las personas y las máquinas. Intervienen el medio ambiente y la totalidad de las dimensiones presentes en cualquier prestación laboral. La organización del trabajo es el resultado del conjunto de reglas y normas que determinan como se ejecuta la producción en la empresa. Desde esta perspectiva es una construcción social, histórica, modificable y cambiante" (Novick, 2000: 126).

Se ha admitido que la búsqueda de nuevas formas de la OT, tiene sus raíces en la empresa. Ella responde a las influencias de los Mercados y las Nuevas Tecnologías, aparecen entonces como los más influyentes factores motivacionales ${ }^{5}$. Las Relaciones de Trabajo si bien no se mencionan en un primer término como generadoras de estas transformaciones, ellas son sujetas de modificaciones, así como también sus propias raíces históricas-sociales y las configuraciones resultantes, influyen en las proposiciones de OT que la empresa aspira y pretende materializar. Aquí juegan lo que llamamos las fuerzas de cambio y las fuerzas de la conservación. Las nuevas propuestas de OT, no sólo consiguen resistencia en quienes aparecen como sus víctimas más visibles, los más próximos al despido por ejemplo, sino que en general se presenta un estado de desasosiego en el conjunto de la fuerza de trabajo. La existencia de un actor colectivo con suficiente liderazgo, es decisiva para que el malestar se convierta en reacciones concretas y no sólo resistencia pasiva.

Con la OT tradicional se conforman los actores de las Relaciones de Trabajo -RT-, así como los mecanismos de intercambio entre las partes. Es por tanto esencial, reconocer que las alteraciones de la organización del trabajo influyen en los comportamientos productivos.

Conviene llamar la atención que históricamente, los más frecuentes estimulantes de los conflictos laborales se ubican en lo económico y/o lo político. En los términos de Zapata (1986:15) ".. la huelga como producto del deterioro del nivel de vida de los trabajadores" por un lado y por el otro: "la huelga como medio para la puesta en práctica de planteamientos ideológico-partidistas que cuestionan la legitimidad política de determi- 
nados regímenes". La dimensión de la OT, es a nuestro juicio una dimensión económica primariamente, y por otro lado, el conflicto cuando adquiere connotación colectiva siembra semillas de confrontación política. La OT es una dimensión económica, porque las transformaciones que en ella se producen están orientadas a la consecución de más rentabilidad, o al menos de mejorar el negocio, sea en prestar un mejor servicio, dejar a los clientes más satisfechos. Zapata formula el ámbito de la OT de la siguiente manera "la huelga como reflejo de la movilización obrera en su base, que se rebela contra el autoritarismo administrativo, el incremento de los ritmos del trabajo o el uso excesivo de los incentivos a la producción". Movilización y rebelión son connotaciones que brindan aprendizaje político en caso de no tenerse, y sí ya se tiene, implica su práctica.

De las tantas modificaciones que han venido experimentando las nuevas formas de OT, unas de ellas es una mayor recurrencia al trabajo externalizado y al uso de contratistas. No son fenómenos nuevos, sólo que tradicionalmente era una recurrencia ocasional, ahora se han convertido en una característica relevan- te de las nuevas formas organizacionales (Plá, 2002:XII; Amorin, 2004).

En las transformaciones que se vienen manifestando en la organización del trabajo, una consecuencia directa en la potencialidad del conflicto laboral colectivo, deriva de lo que Hyman (1996) identifica como: "Una polarización dentro de la clase obrera que muchos autores caracterizan desde el punto de vista de las relaciones entre centro periferia, o entre los de adentro y los de afuera". Esto se evidencia en el tema de la contratación y subcontratación, y sus consecuencias en la integración o agregación del movimiento de los trabajadores. Lo que se traduce en dividir a los trabajadores, facilitando o creando las condiciones para manifestaciones que hacen emerger los intereses divergentes, entre los trabajadores "de adentro y los de afuera".

Respecto a lo planteado, analicemos un reciente caso de conflictividad laboral presentado en el país, como es el conflicto de los trabajadores de la Siderúrgica del Orinoco -Sidor-, organizados en el Sindicato Único de Trabajadores de la Industria Siderúrgica y sus Similares -SUTISS- ${ }^{6}$. Esta huelga se inició el pasado 22 de Abril y se mantuvo por 19 días.

lación de los productores y el mercado, ya sea el de proveedores o de clientes; entonces al final es el Mercado quién se constituye en eje de la transformación que pueda plantearse en la OT. Así pudimos constatarlo en investigación realizada en el sector automotriz y autopartista (Iranzo et al, 1997).

6 Desde fines de la década de los setenta funcionó en la convención colectiva siderúrgica una comisión de arbitraje que atendía las diferencias entre las partes, pero transcurridos pocos años después de la privatización -que se concretó en 1997- este mecanismo se diluyó a fines de la década de los noventa. Contribuyeron igualmente a su inoperancia, las diferencias políticas que resultaron de la división de la Causa $\mathrm{R}$, fuerza dominante por muchos años en este sindicato (Entrevista realizada en noviembre 2004, a Napoleón Goizueta, Ex Presidente de la Comisión de Arbitraje). 
Este sindicato agrupa a 4.000 de los 5.700 trabajadores de la siderúrgica. El tema que interesa destacar, es la relación entre los trabajadores de la nómina de la Siderúrgica del Orinoco -Sidor-y afiliados al Sindicato Único de Trabajadores de la Industria Siderúrgica y sus Similares -Sutiss-, y la reacción de los trabajadores de las empresas contratistas, cuyo número va desde 5 mil hasta 9 mil, dependiendo de la fuente, y no afiliados al sindicato Sutiss. Los trabajadores de las contratas -periféricos-, en la medida que el conflicto se prolonga y por tanto no reciben ingresos, reaccionan ante el sindicato con oposición, ya que carecen de interés en los asuntos centrales del conflicto sindical, con reivindicaciones que no sienten que tengan vinculación con su situación laboral. Por ejemplo, el caso de la no implementación del Programa de Participación Laboral -PPL-por parte del Estado. Al implementarse tendrá repercusión directa sobre los trabajadores de Sutiss, pero esta reivindicación no guarda relación con los trabajadores de las contratistas. El PPL tiene sus antecedentes en el hecho de que al privatizarse Sidor en $1997^{7}$, la ley de privatización planteó un determinado porcentaje accionario para los trabajadores. El Estado quedó de custodio de esta parte del capital -20\%-, y luego por problemas financieros de la empresa, el Estado incrementó su participación, llegando al 30\%. Pero, transcurridos más de seis años este programa no se implementó y cuando los trabajadores reclaman los dividendos de esta porción del capital, estos ya habían sido cobrados por el Estado, como el custodio de estas acciones $^{8}$. De aquí que la Empresa argumente que no debía nada a los trabajadores, a quienes ya había pagado sus utilidades en tantos trabajadores, es decir lo establecido en la convención colectiva ${ }^{9}$.

Hay que tener presente que los trabajadores de una empresa central y los de las contratistas, sí pueden identificar intereses

Esta empresa fue privatizada en diciembre de 1997, adquirida por el Consorcio Amazonia, liderizado por la transnacional Techint, accionista mayoritario del grupo. Amazonia concentra $59.7 \%$ de las acciones de SIDOR. Está formado por Hylsamex (México), Tamsa (México), Siderar (Argentina), Usiminas (Brasil) y Sivensa (Venezuela), empresas siderúrgicas líderes, que concentran el $25 \%$ de la producción de acero líquido en Latinoamérica, y emplean a más de 35 mil personas en forma directa (Sidor, 2004).

8 Ciertamente, una consecuencia de la huelga, ha sido que el Estado al fin haya empezado la implementación de este traspaso de acciones. Se trata de más de 3.5 millones de acciones, que representan el $20 \%$ del total accionario de la empresa. En principio son elegibles para la adquisición de las acciones los 13.655 trabajadores que formaban parte de la empresa al momento de la privatización, sin embargo la aspiración sindical es que además se de oportunidad para quienes están actualmente en nómina. El Universal 18-5-2004.

9 La Empresa emite comunicado que indica "Sidor no tiene deuda alguna con sus trabajadores por concepto de utilidades, porque canceló un monto superior a los establecido por la Ley Orgánica del Trabajo, que es el 15\% de los beneficios líquidos obtenidos por la empresa en el ejercicio anual". Igualmente indica "que no es responsable de la implementación del Programa de Participación Laboral, tarea que le compete al Estado, quien ya anunció su decisión de ejecutarlo." El Nacional del 7-5-2004, pag. B-20. 
convergentes, es decir comunes. Pero eso pasa por aproximarse, por organizarse en una misma entidad sindical, o al menos en casos de distintas organizaciones, establecer mecanismos de intercambio o coordinación. Los sindicatos de la rama $y / 0$ las vinculaciones con una misma central o federación sindical son herramientas para estos casos. Los intereses más globales, los de la clase trabajadora ofrecen un primer ámbito. Pero la cotidianidad en los espacios productivos, exige asuntos de coincidencia y acción gremial más sentidos para los trabajadores. Cuando los diferenciales de ingresos $y$ de condiciones de trabajo entre aquellos percibidos por los trabajadores de la empresa principal y los de las contratistas son muy distantes, estamos en presencia de una situación que favorece más bien la fractura del colectivo laboral.

Uno de los asuntos más sentidos por el colectivo de los trabajadores, aunque más para los llamados periféricos es el de la Seguridad Industrial, sin embargo es llamativo la postergación de este tema ${ }^{10}$. El año pasado ocurrieron 5 accidentes fatales en Sidor, todos trabajadores de las contratas. Se trata de dos administraciones de las condiciones de trabajo, así como de la prevención de los accidentes y riesgos industriales. Una con un personal más entrenado, más protegido, más cercano al concepto de capital humano, y la otra administración en donde hay más desprotección, más trabajo eventual, más incertidumbre, y en muchos casos situaciones de precariedad.
Lo que revela la desigualdad entre los trabajadores de Sidor y los de las contratas. Las reivindicaciones para alcanzar una mejor seguridad industrial en las instalaciones de Sidor, sí representan una meta atractiva para el colectivo global de trabajadores que ahí convergen diariamente. Sutiss anuncia la inclusión de por lo menos 4 mil de los trabajadores de las empresas contratistas en la convención colectiva a discutirse al vencimiento del actual. Están por verse las estrategias para lograrlo. De hacerlo sería un hecho equivalente a las acciones sindicales de los trabajadores petroleros en el convenio colectivo de 1960, en donde lograron extender los beneficios contractuales a los trabajadores de las contratistas. Realmente un hito en la historia de las acciones sindicales del país ${ }^{11}$. Esta es una respuesta concreta en el ámbito de las Relaciones de Trabajo, a los cambios en la OT.

Una empresa de las Ilamadas básicas, como es quién produce acero, tiene al momento de una situación de parálisis efectos multiplicadores, por los encadenamientos productivos. Ese es el caso de los conflictos en Sidor. Los sectores metalúrgico, de fabricación de envases, automotriz, autopartistas, construcción, aparecen entre los más vulnerables. La opción de recurrir al mercado externo, vía importaciones de acero no es una opción que pueda implementarse rápidamente con un producto que tiene alta demanda internacional (Bortesi, 2004:3). Esto hace el conflicto mucho más impactante, pero

10 Entrevista a Eleuterio -"Tello"- Benitez, ex sindicalista de SUTISS y Abogado Laboral de trabajadores de esta empresa, en entrevista del 13 Mayo de 2004. 
al mismo tiempo más vulnerable por los intereses en juego.

El otro fenómeno de las nueva formas de organizar el trabajo y su vulnerabilidad ante los conflictos colectivos, son los llamados procesos "justo a tiempo" que implican no inventarios, o su drástica reducción comparados con el enfoque productivo tradicional de acumulación de inventarios ${ }^{12}$.

Otra visión y experiencia, es aquella que busca que los trabajadores de las nuevas formas de organización del trabajo, les llegue el impacto de las conquistas alcanzadas por los trabajadores de la organización del trabajo tradicional. Tal es la experiencia que se observa en el ámbito de los trabajadores de la prensa en línea y los de la prensa impresa. El colectivo mayor son estos últimos, pero se vienen creando versiones en línea -on linede los mismos periódicos impresos, con una figura jurídica diferenciada, pero trabajando con la misma línea editorial del impreso. ¿Qué ha ocurrido? Los trabajadores de ambas formas de organización del trabajo, participan de las mismas organizaciones sindicales, y la plataforma de acción es de carácter integral (Iturraspe, 2000; Iturraspe y White, 2003). Es evidentemente una respuesta asertiva desde el lado del actor sindical.

\section{La Organización del Trabajo y los Actores Laborales. La constitución y la permanencia del Actor Colectivo}

El eje de la acción en las relaciones laborales descansa en la posibilidad de que los trabajadores se constituyan en actores colectivos. Incluso vale decir lo mismo, cuando se analizan otros ámbitos de la vida de una sociedad. Por ejemplo los vecinos y su accionar en la acción comunitaria, descansa en la constitución de actor colectivo. En las Relaciones de Trabajo la figura del actor colectivo represen-

11 Los últimos treinta años se caracterizaron por una lucha constante de la gerencia petrolera, antes y después de la nacionalización, por eliminar este beneficio. De hecho, ya lo venían logrando en algunos segmentos. Pero ha sido la debacle laboral petrolera de los últimos dos años -2003 y 2004-, la que ha servido para minimizar esta importante conquista, con la cooperativización de las actividades originalmente realizadas por contratistas y sus trabajadores. Se trata de la sustitución de éstos por asociaciones cooperativas, formadas por los propios trabajadores, pero que al mismo tiempo ello implica la no aplicación de la legislación del trabajo. El propio Ministro de Energía y Minas, anunció que en PDVSA "Cooperativas asumirán labores de transporte, servicios, mantenimiento, alimentación, dotación de uniformes, herramientas, trabajos menores" (EI Universal 20-4-03). Igualmente el Ministro informa "Como se recordará, a partir de diciembre de 2002, la empresa PDVSA puso en operación una flota de gandolas que eran propiedad de su filial Deltaven. Estos vehículos se encontraban en desuso por presentar distintas averías. Algunas de estas unidades se repotenciaron para luego ser entregadas, por el Presidente de la República Hugo Chávez, a cooperativas de transporte de hidrocarburos".

12 Es frecuente identificar la acumulación de inventarios, de productos terminados así como de insumos con la caracterización taylorfordista, sin embargo, aún sin cubrir los supuestos organizacionales de esta forma de OT, ha sido frecuente operar con inventarios que proveen a la organización productiva de seguridades, y una de ellas es ante las contingencias derivadas de las Relaciones de Trabajo. 
tativo de los trabajadores, es una condición sine qua non para poder enfrentar el poder patronal. Pero para llegar a ser actor colectivo, el proceso implica una construcción social y política.

Constituirse en actor colectivo demanda que se identifiquen intereses y solidaridades comunes en un conjunto de individuos. El fenómeno es relativamente materializable cuando las personas trabajan en un mismo espacio, para un mismo empleador, para elaborar el mismo producto o servicio. También cuando desarrollan una identidad de oficio o profesión. Pero se dificulta cuando ocurren diversos procesos que ya no permiten la concentración, el encuentro físico y afectivo; así como tampoco se identifica claramente un patrono común; o los oficios se desdibujan. La fragmentación y desconcentración productiva trae aparejada estas consecuencias.

Las nuevas formas de OT tienen en común que expulsan fuerza de trabajo de lo que anteriormente en este documento llamamos el centro. Una preocupación a tener presente, es si todo los expulsados -que pasan a la periferia- son susceptibles de convertirse en actores colectivos, para mantenerse o reinsertarse en el funcionamiento de las relaciones de trabajo. De no ser así, tiene valor analítico y social, la posibilidad de la conversión de actores para otras dimensiones del funcionamiento social. Por ejemplo, los despedidos de PDVSA, de actores laborales y políticos por supuesto, devinieron muchos de ellos primariamente en activos actores políticos en el marco de la sociedad civil. Sus intentos en persistir como actores laborales -vía Unapetrol-, han encontrado escollos aún no resueltos ${ }^{13}$.

Otro fenómeno frecuente es la salida de trabajadores de aquellas organizaciones empleadoras, en donde para motivar y hacer estimulante la reducción de personal, se ofrece al trabajador saliente de una atractiva y generosa provisión monetaria -popularmente conocida como la "cajita feliz"-, la que puede convertir al trabajador que ha acumulado varios años de prestaciones en un pequeño empresario, y que luego devenga en un activo socio de los gremios empresariales; de ser integrante de un actor laboral -asalariado-, pasa a actor laboral empresarial. Otra opción es devenir en un ciudadano, que minimiza sus lazos con el movimiento de los trabajadores, y asume una posición pasiva en torno a los problemas y reivindicaciones de la clase trabajadora.

El problema de la expulsión de trabajadores de los centros productivos, y que los mismos quedan atados de alguna manera al centro expulsor, ya que van a prestar servicios a empresas relacionadas como contratistas, proveedores $y$ clientes, es que en situaciones de un mercado laboral deprimido, y dado el hecho que los nuevos patronos y las condiciones de trabajo que imperan en la nueva situación desmejoran las existentes, in-

13 Aunque el paro petrolero de diciembre de 2002-enero de 2003 no tiene vinculación con los fenómenos de los cambios en la Organización del Trabajo, se puede leer un análisis del mismo en Lucena (2002). 
cluso en no pocos casos llegando a situaciones de precarización, coloca a estos trabajadores a añorar su viejo empleo, del cual fue expulsado. Esto no excluye la esperanza e ilusión de un retorno al mismo, incluso en condiciones igualmente desventajosas, con respecto a aquellas que disfrutaba al momento de expulsión.

Los actores constituidos, es decir los sindicatos establecidos, tienen ante sí una situación dilemática ante el proceso de reestructuración productiva y la expulsión de trabajadores de los centros productivos. Se trata de la preservación de los vínculos afectivos y solidarios con estos trabajadores. Es un fenómeno importante, por encontrarnos con procesos continuos y sostenidos de reducción de la fuerza de trabajo, y el replanteamiento de la organización empresarial, apuntando a nuevas formas, en donde la organización tradicional centralizada y con crecimientos vertical y horizontalmente, da paso a diversidad de otras formas, todas caracterizadas por el achicamiento del número de trabajadores.

Se plantea si al sindicalismo, actor constituido, le toca ser el organizador de los expulsados, sea por abrir un campo o espacio en su organización, o contribuir a la creación de otras entidades colectivas. En el primer caso, la vía de pasar de organización de empresa a organización de rama y de profesionales, en donde la figura del desempleado, o del laborante inestable, encuentra en la organización de este tipo un espacio de solidaridad y encuentro. Lo otro es prestarse al apoyo a las nuevas organizaciones, que resulten del accionar de los nuevos actores, por ejemplo los pensionados, los jubilados. Son ex-trabajadores que construyeron la- zos afectivos con quienes fueron sus compañeros de trabajo. Vemos como las organizaciones de jubilados y pensionados, generalmente se construyen al margen y diferenciadas de las organizaciones sindicales de donde proceden los trabajadores.

En las elecciones sindicales de este nuevo siglo, ha venido tomando cuerpo el planteamiento de tener presente a los jubilados y pensionados con derecho a voto para la elección de los cargos en las estructuras sindicales. Se debatió en el 2001 en las pasadas elecciones sindicales. Reina Sequera, para entonces candidata independiente a presidir la CTV, manifestó "Creo que no debemos olvidar el derecho al voto de los pensionados y jubilados "(El Nacional, 29-8-01, D1). Se vuelve a debatir actualmente a propósito de las elecciones sindicales a celebrarse en el 2005.

El tener integrado a los expulsados de la condición de asalariados de las empresas centrales, que luego pasan a la condición de periféricos, por trabajar en las empresas contratistas, es una aspiración que bien pudiera considerarse a los fines del derecho a voto en las elecciones sindicales. Esto obligaría a la dirigencia a tener una plataforma reivindicativa que no se concentra sólo en los centrales, y que contrarrestaría los efectos perniciosos y precarizadores de muchos de los cambios en la OT.

\section{Conflictos laborales y la comunidad}

Un ámbito que ha devenido frecuente en los conflictos laborales, es llevarlo a la calle (Sappia, 2002; Ermida, 
1999). Es decir que no se conforman los promotores del conflicto en la acción directa sobre la otra parte, sino que también es significativo hacerlo trascender a la opinión pública. La connotación de llevarlo a la calle, a la opinión pública, encuentra sus canales además en los medios de comunicación social ${ }^{14}$. El propósito de esta estrategia no es ajeno al conflicto mismo, se trata de que la presión de la comunidad y sus posiciones en torno al conflicto, puedan ejercer algún efecto. Sensibilizar con su crítica y posición, a la otra parte. He aquí otro elemento en donde la frontera del conflicto laboral con el ámbito político se hace nuevamente muy tenue, ya que al llevar a la calle el conflicto laboral, se coloca la acción de los promotores cerca de las comunidades y los ciudadanos, intercambio que facilita y expone a los huelguistas cerca de situaciones que rayan en la alteración de la paz social.

La relación con la comunidad es sensible cuando el conflicto procede de empresas de servicio. Los conflictos de los transportistas de personas en las urbes, que generalmente se traducen en aumentos de las tarifas para los usuarios, conllevan una situación de riesgo para los huelguistas, ante la reacción de las comunidades. De paso hay que destacar, que en este ámbito de la prestación de servicios, en muchas ciudades predominan los cuentapropistas, es decir, los transportistas dueños de sus unidades de transporte de pasajeros. Pero es un sector que ha devenido en un activo actor colectivo, con una notable capacidad de presionar a las autoridades. Es, dentro de la informalidad, uno de los segmentos más organizado ${ }^{15}$.

Los cambios en la OT alcanzan dimensiones complejas que facilitan su impacto en las comunidades cuando implican reducción significativa de la fuerza de trabajo, así como cuando los cambios en cuestión llegan al público o a la comunidad afectándoles, tal es el caso de las tarifas de servicios. De lo contrario, estos cambios discurren erosionando conquistas históricas de los trabajadores, así como su patrimonio. Que en buena medida es el drama de las transformaciones de la OT, que se suceden en ámbitos de inexistencia o existencia de débiles actores laborales representativos de los trabajadores.

\section{Las asociaciones cooperativas como nuevos actores laborales}

Las cooperativas son una vieja forma de asociatividad y empresarialidad que data de siglos. En Venezuela se observan desde el primer tercio del siglo pasado, y de manera paulatina fueron difundiéndose en diversas partes del país. Su mayor desarrollo se alcanzó en el Estado Lara y los estados andinos. Para el año

14 En el conflicto de Sidor-Sutiss, que hemos analizado en otras dimensiones en el presente artículo, expresa esta característica en el hecho que los trabajadores promovieron marchas en las principales calles de Ciudad Guayana, y por otro lado la empresa, emitía comunicados de alta visibilidad en la prensa local y regional, a medida que el conflicto avanzaba.

15 No hay duda que los transportistas han devenido en efectivos actores laborales, para luchar por sus reivindicaciones, tarifas más que todo. 
2000, en el país se contabilizaban dos mil quinientas organizaciones cooperativas, registradas en la Superintendencia respectiva. Para fines del 2004, este número alcanzaba las cuarenta mil, según Carlos Molina el Superintendente Nacional de Cooperativas (Sunacoop, 2004).

Para entender este fenómeno del incremento explosivo de estas organizaciones, evidentemente lo que se denomina un "boom", y de hecho para entender la vinculación que esto tiene con las nuevas formas de organización del trabajo, conviene considerar los siguientes aspectos. Primero, existe una política estatal de fomento de las asociaciones cooperativas (Presidencia de la República, 2001; Barrios, 2003). Además con motivo del paro nacional de diciembre 2002 y enero 2003, quedó desarticulado el sector petrolero por los despidos de diez y nueve mil trabajadores, y la ruptura de la empresa estatal PDVSA con la mayor parte de las empresas contratistas, por lo que el gobierno optó por el fomento de las asociaciones cooperativas, como opción para contratar servicios y actividades realizadas anteriormente con asalariados de la propia empresa petrolera y concontratistas -la vieja externalización- empresariales. Una segunda gran razón del "boom" del cooperativismo, es el aprovechamiento que las empresas industriales $y$ de servicio hacen de esta forma organizativo, ya que ella supone diversas ventajas empresariales, como son ventajas tributarias y laborales, aparte de la disposición gubernamental para proveer financiamiento a estas nuevas cooperativas. El artículo 34 indica claramente que los asociados "no tienen vinculo de dependencia con la cooperativa y los anticipos no tienen condición de salario".."no estarán sujetos a la legislación laboral' (Presidencia de la República, 2001).

Evidentemente que la sustitución de trabajadores asalariados por cooperativistas, en un abierto fraude laboral, abona a favor de disminuir la conflictividad. La OIT (Citado por Barrios 2003:9) en su 90를 Conferencia -junio de 2002- advirtió de este peligro para los trabajadores, aprobando un texto en donde indicaba que las políticas nacionales deberían: "..velar porque no se puedan crear o utilizar cooperativas para evadir la legislación del trabajo, ni ello sirva para establecer relaciones de trabajo encubiertas y luchar contra las seudocooperativas, que violan los derechos de los trabajadores, velando por que la legislación del trabajo se aplique en todas las empresas".

Los trabajadores que pasan de asalariados a cooperativistas, ya no son sujetos de la organización sindical. De hecho han coincidido el gobierno y las empresas en promover esta opción organizacional. En el ámbito gubernamental, se manifiestan preocupaciones, porque con seudocoperativismo, hay dificultades para la determinación de responsabilidades en cuanto a la seguridad social y las condiciones y medio ambiente de trabajo -entrevista con J Piccone, Director Nacional de Inspección del Ministerio del Trabajo, 8 Julio 2004-.

\section{Conclusiones}

Todo conflicto laboral abierto encierra larvadamente semillas políticas. Es una prueba de fuerza. La derrota o la victoria cuentan en el juicio que los seguidores hagan de los líderes, así también la 
contraparte. Los seguidores fortalecerán la adhesión al liderazgo en el conflicto, si el resultado muestra un balance favorable. La contraparte asumirá una posición de respeto, revisará sus estrategias o agudizará la tirantez. En la medida que se prolonga una acción huelgaria, dejará ver más claramente los ribetes políticos de la confrontación de fondo, entre los intereses del capital y del trabajo. Generalmente un conflicto deja huellas, heridas, incluso traumas.

Por ejemplo, en cuanto al conflicto de Sidor y Sutiss analizado desde la perspectiva de la relación entre trabajadores centrales y trabajadores periféricos, tiene en su dimensión política, que concluido el mismo, surgen los análisis y las acusaciones. Ello lleva a preguntarse ¿qué se logró? ¿a cuál precio? Los trabajadores y la empresa se plantean ¿ganamos o perdimos $?^{16}$. El ejercicio que se plantea en la confrontación, no sólo debe evaluarse en la coyuntura y el hecho inmediato, también hay dimensiones ligadas al aprendizaje de los actores, tanto en su liderazgo como en las bases. En los conflictos se hacen demostraciones de autonomía, de emprender caminos propios. Esto es relevante en un sindicalismo fuertemente tutelado por el Estado. "Reconocíamos allí el dilema histórico del sindicalismo latinoamericano, es decir, aquel de su definición frente al Estado y al sistema político" (Zapata, 1986:169). En todo conflicto laboral estos son ejercicios ineludibles.
Hay que advertir que el conflicto puede tener claramente motivaciones económicas y no vérsele por ningún lado motivaciones políticas, pero es del interés de la contraparte destacar estas motivaciones para quitar sustancia a los reclamos económicos. En el ámbito de la administración pública suele ocurrir, que los demandantes que reclaman por ejemplo sueldos atrasados o deudas laborales, son soslayados por endilgarles a sus reclamos motivaciones primariamente políticas, por ser instituciones lideradas por dirigentes contrarios a los que conducen las instituciones sometidas al reclamo.

Este tema de los conflictos laborales y la organización del trabajo es de permanente relevancia. En la búsqueda empresarial de nuevas formas organizativas, siempre está presente la inquietud fundamental, de encontrar salidas que contrarresten el poder de los trabajadores. De aquí que el dinamismo que tienen las renovadas formas organizativas para producir, que se traducen en la localización de novísimas formas de organizar el trabajo o en algunos casos, no tan novedosas, pero sí esquemas y formas de poca difusión, y que el capital redescubre en ellas importantes ventajas para la explotación del trabajo. Es por esto que la investigación laboral, se ve obligada a mirar detenidamente, las reales motivaciones que subyacen en las formas "modernas" de organizar el trabajo y la situación del trabajo dependiente. Estimamos que

16 Los diversos grupos de opinión que conforman la directiva levantan posiciones triunfalistas o acusadoras. La empresa por su parte fortalece sus posturas, destacando su apego a la legalidad por encima de todo, lo que en el fondo también es una posición política. 
en la externalización, la contratación, el trabajo cooperativo en tanto esfuerzo simulador de las relaciones de trabajo, se advierten manifestaciones que atentan contra una herramienta importante en las Relaciones de Trabajo, como es la capacidad conflictiva de los trabajadores.

\section{Referencias Bibliográficas}

\section{Alfonso Guzmán, Rafael (1967), Estudio Ana- lítico de la Ley del Trabajo Venezo- lana. Tomo Dos, Edit. UCV.}

Amorin, Ronald (2004), "El derecho social frente a las nuevas formas de trabajo" en Gaceta Laboral, Vol.10, No.2, pp. 229-234.

Barrios, Froilán (2003), Cooperativismo y flexibilización laboral. Folleto Edit. por el Frente Constituyente de los Trabajadores, Corriente sindical Nacional; Caracas.

Bortesi, Bruno (2004), "Corresponde al gobierno solventar crisis de Sidor. La industria manufacturera apenas tiene inventarios de acero para operar unos días", Presidente de GE Andina. Suplemento Industrial de la CIEC en EI Carabobeño, Mayo.

Coller, Xavier (1997), "La empresa flexible. Estudio sociológico del impacto de la flexibilidad en el proceso de trabajo" Edit. C.I.S. -Centro de Investigaciones Sociológicas-, Colección Monografías, No. 155; Madrid.

Córdova, Efrén (2004), "La Ley Orgánica Procesal del Trabajo a la luz de la legislación comparada" en Gaceta Laboral, Vol.10, No. 1, pp. 5-20.

El Nacional del 7-5-2004, Pág. B-20. Comunicado de la dirección de la empresa Sidor.

El Nacional, del 29-8-01, Pág. D1, Declaraciones de Reina Sequera.
El Universal, Declaraciones de Rafael Sánchez M., Presidente de la Corporación Venezolana de Guayana, en: http://buscador.eluniversal.com/ 2004/05/13/eco_ ava_13A460937.shtm

El Universal, Declaraciones de Rafael Ramírez, Ministro de Energía y Minas en: http://buscador.eluniversal.com/ 2003/04/20/eco_art_20112CC.shtml

Ermida, Oscar (1999), "La flexibilización de la huelga" Cuadernos de Fundación Derecho del Trabajo y de la Seguridad Social, Edit. Fundación de la cultura universitaria, Montevideo.

Hernández, Marcela (2003), "Subjetividad y cultura en la toma de decisiones empresariales" Edit UAA y Plaza y Valdez, México.

Hyman, Richard (1996), "Los sindicatos y la desarticulación de la clase obrera" en Revista Latinoamericana de Estudios del Trabajo, Edit. ALAST, Año 2, No.4; Sao Paulo, p.9-28.

Iranzo, Consuelo (Coord.); Lucena, Héctor; Sandoval, Fausto y Betencourt, Luisa (1997), "Relaciones Laborales al Desnudo" Edit. Cendes-Venezuela Competitiva; Caracas.

Iturraspe, Francisco y White, Aidan (2003), "Estrategias de los trabajadores frente a los medios globales y digitales en América Latina"; Edit. FIP (Federación Internacional de Periodistas); Caracas.

Iturraspe, Francisco (2000), "Democracia y conflicto. Deslegitimación del sistema político venezolano (1958-1998). La ilegalización de la huelga como índice de subdesarrollo político" en Gaceta Laboral, Vol.6, No.3, pp. 347-380.

Lucena, Héctor (2002), "Confrontación y paros nacionales en Venezuela. Exploraciòn preliminar" en Revista Latinoamericana de Estudios del Trabajo Año 8 No. 15, Buenos Aires, pp. 173192. 
Lucena, Héctor (2003), Las Relaciones de Trabajo en el nuevo siglo. Edit. Tropykos, Caracas.

Marx, Karl (1973), "Trabajo Asalariado y Capital" en Marx, K y Engels, Frederick. Obras Escogidas. 3 Tomos, Editorial Progreso, Instituto de Marxismo-Leninismo, Moscú.

Novick, Martha (2000), "La transformación de la organización del trabajo" pp. 123148. En Tratado Latinoamericano de Sociología del Trabajo. E de la Garza -Compilador- Edit. FCE, UAM, El Colegio de México.

Plá Rodríguez, Américo (2002), "Los principios del derecho del trabajo y I a globalización económica" en Análisis Laboral, Lima, Vol. XXVI, No. 302, Agosto pp. IX-XII.

Presidencia de la República Bolivariana de Venezuela (2001), "Decreto con fuerza de ley especial de asociaciones cooperativas", Gaceta Oficial No. 37.285 del 18 dic. 2001.

Romero, Antonio (1997), "Cronología y análisis de las huelgas en Venezuela, 1989-1993" Edit. UCLA-CDCHT, Barquisimeto, pp.146.

Romero, Antonio (2001), "El conflicto laboral en Venezuela. Caso de los servicios públicos esenciales, período 19941998" Edit. UCLA, pp.158.

Sappia, Jorge (2002), Justicia Laboral y medios alternativos de solución de conflictos colectivos e individuales del trabajo. OIT Lima, Doc. 149.

Sidor. Consulta en Internet el 29 de Junio de 2004: http://www.sidor.com.ve/empresa/empresa.htm
Sunacoop. Consulta en Internet el 20 de diciembre de 2004 http://www.sunacoop.gov.ve

Van der Laat, Bernardo (1979), "La huelga y el paro en Costa Rica" Edit. Juricentro, San José.

Velasco, Enrique (1976), "Introducción al derecho del trabajo" Edit. Blume, Barcelona.

Zapata, Francisco (1986), El conflicto sindical en América Latina. Edit. El Colegio de México. México DF.

Zapata, Francisco (2003), La historia del movimiento obrero en América Latina y sus formas de investigación. Pp. 371-391; en De la Garza, E -coordinador- "Tratado Latinoamericano de Sociología del Trabajo" Edit FCE, El Colegio de México, UAM, FLACSO. México.

\section{Entrevistas}

Benítez, Eleuterio -"Tello"- (2004), Ex sindicalista de SUTISS y Abogado Laboral de trabajadores de esta empresa, entrevista del 13 Mayo.

Goizueta, Napoleón (2004), Profesor de Derecho del Trabajo de la Universidad de Carabobo y Ex Presidente de la Comisión de Arbitraje Sidor-Sutiss. Entrevista realizada en noviembre.

Piccone, Johnny (2004), Director Nacional de Inspección del Ministerio del Trabajo, entrevista del 8 de Julio. 
Lucena, Héctor (2003), Las Relaciones de Trabajo en el nuevo siglo. Edit. Tropykos, Caracas.

Marx, Karl (1973), "Trabajo Asalariado y Capital" en Marx, K y Engels, Frederick. Obras Escogidas. 3 Tomos, Editorial Progreso, Instituto de Marxismo-Leninismo, Moscú.

Novick, Martha (2000), "La transformación de la organización del trabajo" pp. 123148. En Tratado Latinoamericano de Sociología del Trabajo. E de la Garza -Compilador- Edit. FCE, UAM, El Colegio de México.

Plá Rodríguez, Américo (2002), "Los principios del derecho del trabajo y I a globalización económica" en Análisis Laboral, Lima, Vol. XXVI, No. 302, Agosto pp. IX-XII.

Presidencia de la República Bolivariana de Venezuela (2001), "Decreto con fuerza de ley especial de asociaciones cooperativas", Gaceta Oficial No. 37.285 del 18 dic. 2001.

Romero, Antonio (1997), "Cronología y análisis de las huelgas en Venezuela, 1989-1993" Edit. UCLA-CDCHT, Barquisimeto, pp.146.

Romero, Antonio (2001), "El conflicto laboral en Venezuela. Caso de los servicios públicos esenciales, período 19941998" Edit. UCLA, pp.158.

Sappia, Jorge (2002), Justicia Laboral y medios alternativos de solución de conflictos colectivos e individuales del trabajo. OIT Lima, Doc. 149.

Sidor. Consulta en Internet el 29 de Junio de 2004: http://www.sidor.com.ve/empresa/empresa.htm
Sunacoop. Consulta en Internet el 20 de diciembre de 2004 http://www.sunacoop.gov.ve

Van der Laat, Bernardo (1979), "La huelga y el paro en Costa Rica" Edit. Juricentro, San José.

Velasco, Enrique (1976), "Introducción al derecho del trabajo" Edit. Blume, Barcelona.

Zapata, Francisco (1986), El conflicto sindical en América Latina. Edit. El Colegio de México. México DF.

Zapata, Francisco (2003), La historia del movimiento obrero en América Latina y sus formas de investigación. Pp. 371-391; en De la Garza, E -coordinador- "Tratado Latinoamericano de Sociología del Trabajo" Edit FCE, El Colegio de México, UAM, FLACSO. México.

\section{Entrevistas}

Benítez, Eleuterio -"Tello"- (2004), Ex sindicalista de SUTISS y Abogado Laboral de trabajadores de esta empresa, entrevista del 13 Mayo.

Goizueta, Napoleón (2004), Profesor de Derecho del Trabajo de la Universidad de Carabobo y Ex Presidente de la Comisión de Arbitraje Sidor-Sutiss. Entrevista realizada en noviembre.

Piccone, Johnny (2004), Director Nacional de Inspección del Ministerio del Trabajo, entrevista del 8 de Julio. 